\title{
SHARP VALUES FOR THE CONSTANTS IN THE POLYNOMIAL BOHNENBLUST-HILLE INEQUALITY
}

\author{
P. JIMÉNEZ-RODRÍGUEZ, G.A. MUÑOZ-FERNÁNDEZ, M. MURILLO-ARCILA, \\ AND J.B. SEOANE-SEPÚLVEDA
}

\begin{abstract}
In this paper we prove that the complex polynomial Bohnenblust-Hille constant for 2-homogeneous polynomials in $\mathbb{C}^{2}$ is exactly $\sqrt[4]{\frac{3}{2}}$. We also give the exact value of the real polynomial Bohnenblust-Hille constant for 2-homogeneous polynomials in $\mathbb{R}^{2}$. Finally, we provide lower estimates for the real polynomial Bohnenblust-Hille constant for polynomials in $\mathbb{R}^{2}$ of higher degrees.
\end{abstract}

\section{Preliminaries: What you need to knOW}

Any homogeneous polynomial in $\mathbb{K}^{n}(\mathbb{K}=\mathbb{R}$ or $\mathbb{C}$ ) of degree $m \in \mathbb{N}$ can be written as

$$
P(x)=\sum_{|\alpha|=m} a_{\alpha} x^{\alpha}
$$

where $x=\left(x_{1}, \ldots, x_{n}\right) \in \mathbb{K}^{n}, \alpha=\left(\alpha_{1}, \ldots, \alpha_{n}\right) \in(\mathbb{N} \cup\{0\})^{n},|\alpha|=\alpha_{1}+\cdots+\alpha_{n}, x^{\alpha}=$ $x_{1}^{\alpha_{1}} \cdots x_{n}^{\alpha_{n}}$ and $a_{\alpha} \in \mathbb{K}$. Here, $\mathcal{P}\left({ }^{m} \mathbb{K}^{n}\right)$ stands for the finite dimension linear space of all the homogeneous polynomials of degree $m$ on $\mathbb{K}^{n}$.

If $\|\cdot\|$ is a norm on $\mathbb{K}^{n}$, then the formula

$$
\|P\|:=\sup \left\{|P(x)|: x \in \mathrm{B}_{X}\right\},
$$

for all $P \in \mathcal{P}\left({ }^{m} \mathbb{K}^{n}\right)$, where $\mathrm{B}_{X}$ is the unit ball of the Banach space $X=\left(\mathbb{K}^{n},\|\cdot\|\right)$, defines a norm in $\mathcal{P}\left({ }^{m} \mathbb{K}^{n}\right)$ usually called polynomial norm. The space $\mathcal{P}\left({ }^{m} \mathbb{K}^{n}\right)$ endowed with the polynomial norm induced by $X$ is denoted by $\mathcal{P}\left({ }^{m} X\right)$.

Other norms customarily used in $\mathcal{P}\left({ }^{m} \mathbb{K}^{n}\right)$ besides the polynomial norm are the $\ell_{p}$ norms of the coefficients. Namely, if $P$ is as in (1.1) and $p \geq 1$, then

$$
|P|_{p}:=\left(\sum_{|\alpha|=m}\left|a_{\alpha}\right|^{p}\right)^{\frac{1}{p}}
$$

defines another norm in $\mathcal{P}\left({ }^{m} \mathbb{K}^{n}\right)$. It is important to point out that although the polynomial norm is, most of the times, very difficult to compute, the $\ell_{p}$ norm of the coefficients is fairly easy to obtain. Since $\mathcal{P}\left({ }^{m} \mathbb{K}^{n}\right)$ is finite dimensional, the polynomial norm $\|\cdot\|$ and the $\ell_{p}$ $\operatorname{norm}|\cdot|_{p}(p \geq 1)$ are equivalent, and therefore there exist constants $k(m, n), K(m, n)>0$ such that

$$
k(m, n)|P|_{p} \leq\|P\| \leq K(m, n)|P|_{p},
$$

2010 Mathematics Subject Classification. 46G25, 47L22, 47H60.

Key words and phrases. Bohnenblust-Hille constants, Absolutely summing operators, Quantum Information Theory.

G.A. Muñoz-Fernández and J.B. Seoane-Sepúlveda were supported by MTM2012-34341. M. MurilloArcila was supported by a grant of the FPU program of MEC, and by MTM2013-47093. 
for all $P \in \mathcal{P}\left({ }^{m} \mathbb{K}^{n}\right)$. The latter inequalities may provide a good estimate on $\|P\|$ as long as we know the exact value of the best possible constants $k(m, n)$ and $K(m, n)$ appearing in (1.2).

The problem presented above is an extension of the the well known polynomial Bohnenblust-Hille inequality (polynomial $\mathrm{BH}$ inequality for short). It was proved in [5] that there exists a constant $D_{m}>0$ such that for every $P \in \mathcal{P}\left({ }^{m} \ell_{\infty}^{n}(\mathbb{K})\right)$ we have

$$
|P|_{\frac{2 m}{m+1}} \leq D_{m}\|P\|
$$

where $\ell_{\infty}^{n}(\mathbb{R})$ and $\ell_{\infty}^{n}(\mathbb{C})$ are, respectively, the real and complex versions of $\ell_{\infty}^{n}$. Observe that (1.3) coincides with the first inequality in (1.2) for $p=\frac{2 m}{m+1}$ except for the fact that $D_{m}$ in (1.3) can be chosen in such a way that it is independent from the dimension $n$. Actually Bohnenblust and Hille showed that $\frac{2 m}{m+1}$ is optimal in (1.3) in the sense that for $p<\frac{2 m}{m+1}$, any constant $D$ fitting in the inequality

$$
|P|_{p} \leq D\|P\|
$$

for all $P \in \mathcal{P}\left({ }^{m} \ell_{\infty}^{n}(\mathbb{K})\right)$ depends necessarily on $n$.

The best constants in (1.3) depend considerably on whether we consider the real or the complex version of $\ell_{\infty}^{n}$, which motivates the following definition:

Definition 1.1. The polynomial Bohnenblus-Hille constant for polynomials of degree $m$ is defined as

$$
D_{\mathbb{K}, m}:=\inf \left\{D>0:|P|_{\frac{2 m}{m+1}} \leq D\|P\|, \text { for all } n \in \mathbb{N} \text { and } P \in \mathcal{P}\left({ }^{m} \ell_{\infty}^{n}(\mathbb{K})\right)\right\} .
$$

If we restrict attention to a certain subset $E$ of $\mathcal{P}\left({ }^{m} \ell_{\infty}^{n}(\mathbb{K})\right)$ for some $n \in \mathbb{N}$, then we define

$$
D_{\mathbb{K}, m}(E):=\inf \left\{D>0:|P|_{\frac{2 m}{m+1}} \leq D\|P\| \text { for all } P \in E\right\} .
$$

For simplicity we will often use the notation $D_{\mathbb{K}, m}(n)$ instead of $D_{\mathbb{K}, m}\left(\mathcal{P}\left({ }^{m} \ell_{\infty}^{n}(\mathbb{K})\right)\right)$. Note that

for all $m, n \in \mathbb{N}$.

$$
1 \leq D_{\mathbb{K}, m}(n) \leq D_{\mathbb{K}, m}
$$

A good idea of the asymptotic growth of the constants $D_{\mathbb{K}, m}$ and $D_{\mathbb{K}, m}(n)$ is provided by the following definition:

Definition 1.2. The asymptotic hypercontractivity constant of the polynomial BH inequality is

$$
H_{\mathbb{K}, \infty}:=\limsup _{m} \sqrt[m]{D_{\mathbb{K}, m}}
$$

Similarly, if we restrict attention to polynomials in $n$ variables then we define

$$
H_{\mathbb{K}, \infty}(n):=\limsup _{m} \sqrt[m]{D_{\mathbb{K}, m}(n)} .
$$

Of course $1 \leq H_{\mathbb{K}, \infty}(n) \leq H_{\mathbb{K}, \infty}$, for all $n \in \mathbb{N}$.

It was shown in [10] that the complex polynomial Bohnenblust-Hille inequality is, at most, hypercontractive. In [2] the estimate on $D_{\mathbb{C}, m}$ was improved. In fact the authors show that for every $B>1$ there exists $A>0$ such that $D_{\mathbb{C}, m} \leq A e^{B \sqrt{m \log m}}$, from which it follows that $H_{\mathbb{C}, \infty}(n)=H_{\mathbb{C}, \infty}=1$, for all $n \in \mathbb{N}$. For the real case, it has been recently proved in [7] that $H_{\mathbb{R}, \infty}=2$. However, not many exact values of $D_{\mathbb{K}, m}(n)$ are known so far. This paper is devoted to calculate, explicitly or numerically some values of these constants. 
This paper is arranged in two main sections. In Section 2, we employ some results on the geometry of spaces of polynomials in order to provide the exact value of $D_{\mathbb{C}, 2}(2)$. In Section 3 we use a similar technique to find the exact value of $D_{\mathbb{R}, 2}(2)$. We also provide lower estimates for $D_{\mathbb{R}, m}(2)$ and $H_{\mathbb{R}, \infty}(2)$ by means of numerical calculus.

The polynomial Bohnenblust-Hille inequality has important applications in different fields of Mathematics and Physics and has been studied in depth by many authors since a multilinear version of the Bohnenblust-Hille inequality was proved in 1931 (see [2-6,9,11-17,20-27]) and the references therein.

\section{The exact value of $D_{\mathbb{C}, 2}(2)$}

Throughout this section we will often identify any two-variable polynomial $a z^{2}+b w z+c w^{2}$ or any one-variable polynomial $a \lambda^{2}+b \lambda+c$, for $a, b, c \in \mathbb{K}$, with the vector $(a, b, c) \in \mathbb{K}^{3}$. Also, we use the standard notation $\left\|a z^{2}+b w z+c w^{2}\right\|_{\mathbb{D}}$ for the supremum of $\left|a z^{2}+b w z+c w^{2}\right|$ for $z, w$ in the unit disk $\mathbb{D}$ of $\mathbb{C}$. Similarly, $\left\|a \lambda^{2}+b \lambda+c\right\|_{\mathbb{D}}$ stands for the supremum of $\left|a \lambda^{2}+b \lambda+c\right|$ for $\lambda \in \mathbb{D}$. Observe that

$$
\left\|a z^{2}+b w z+c w^{2}\right\|_{\mathbb{D}}=\left\|a \lambda^{2}+b \lambda+c\right\|_{\mathbb{D}}=\max _{|\lambda|=1}\left|a \lambda^{2}+b \lambda+c\right|,
$$

being the last of the latter equalities due to the Maximum Modulus Principle.

The main result of this section depends upon the following lemma, which is of independent interest.

Lemma 2.1. Let $a, b, c \in \mathbb{C}$. There exist $a^{\prime}, b^{\prime}, c^{\prime} \in \mathbb{R}$ such that

$$
\left\|a z^{2}+b w z+c w^{2}\right\|_{\mathbb{D}} \geq\left\|a^{\prime} z^{2}+b^{\prime} z w+c^{\prime} w^{2}\right\|_{\mathbb{D}} \quad \text { and } \quad\|(a, b, c)\|_{\frac{4}{3}}=\left\|\left(a^{\prime}, b^{\prime}, c^{\prime}\right)\right\|_{\frac{4}{3}} .
$$

Proof. If we perform the change of variables

$$
z \mapsto z e^{-\frac{i \arg (a)}{2}} \text { and } w \mapsto w e^{-\frac{i \arg (c)}{2}},
$$

in $\left\|a z^{2}+b z w+c w^{2}\right\|_{\mathbb{D}}$, we can assume (without loss of generality) that $a, c \geq 0$. We can also assume that $a \geq c$ by swapping $z$ and $w$. We have:

$$
\begin{aligned}
\left|a \lambda^{2}+b \lambda+c\right|^{2} & =\left(a \lambda^{2}+b \lambda+c\right)\left(a \overline{\lambda^{2}}+\overline{b \lambda}+c\right) \\
& =a^{2}+a c \lambda^{2}+a \bar{b} \lambda+a c \overline{\lambda^{2}}+c^{2}+c \overline{b \lambda}+a b \bar{\lambda}+b c \lambda+|b|^{2} \\
& =a^{2}+c^{2}+|b|^{2}+2\left[a c \operatorname{Re}\left(\lambda^{2}\right)+a \operatorname{Re}(\bar{b} \lambda)+c \operatorname{Re}(b \lambda)\right] \\
& =a^{2}+c^{2}+|b|^{2}+2\left[a c \operatorname{Re}\left(\lambda^{2}\right)+(a+c) \operatorname{Re}(b) \operatorname{Re}(\lambda)+(a-c) \operatorname{Im}(b) \operatorname{Im}(\lambda)\right] .
\end{aligned}
$$

Similarly, if $a^{\prime}, b^{\prime}, c^{\prime}$ are real numbers, then:

$$
\left|a^{\prime} \lambda^{2}+b^{\prime} \lambda+c^{\prime}\right|^{2}=a^{\prime 2}+c^{\prime 2}+\left|b^{\prime}\right|^{2}+2\left[a^{\prime} c^{\prime} \operatorname{Re}\left(\lambda^{2}\right)+\left(a^{\prime}+c^{\prime}\right) b^{\prime} \operatorname{Re}(\lambda)\right] .
$$

(1) Assume first $a \geq c \geq|b|$. Then, choose

$$
a^{\prime}=\frac{\left(c^{\frac{4}{3}}+|b|^{\frac{4}{3}}\right)^{\frac{3}{4}}}{2^{\frac{3}{4}}}, c^{\prime}=-a^{\prime}, b^{\prime}=a .
$$

Then, $\|(a, b, c)\|_{\frac{4}{3}}=\left\|\left(a^{\prime}, b^{\prime}, c^{\prime}\right)\right\|_{\frac{4}{3}}$. On the other hand,

$$
a^{\prime 2}+c^{\prime 2}+\left|b^{\prime}\right|^{2}+2\left[a^{\prime} c^{\prime} \operatorname{Re}\left(\lambda^{2}\right)+\left(a^{\prime}+c^{\prime}\right) b^{\prime} \operatorname{Re}(\lambda)\right]=2 a^{\prime 2}+a^{2}-2 a^{\prime 2} \operatorname{Re}\left(\lambda^{2}\right),
$$

so that it is easy to see

$$
\left\|\left(a^{\prime}, b^{\prime}, c^{\prime}\right)\right\|_{\mathbb{D}}^{2}=4 a^{\prime 2}+a^{2}=\sqrt{2}\left(c^{\frac{4}{3}}+|b|^{\frac{4}{3}}\right)^{\frac{3}{2}}+a^{2} .
$$


Also, giving the value $\lambda=1($ if $\operatorname{Re}(b) \geq 0)$ or $\lambda=-1$ (if $\operatorname{Re}(b) \leq 0)$, we can see that

$$
\left\|a z^{2}+c w^{2}+b z w\right\|_{\mathbb{D}}^{2} \geq a^{2}+c^{2}+|b|^{2}+2 a c .
$$

Now, we want

$$
\sqrt{2}\left(c^{\frac{4}{3}}+|b|^{\frac{4}{3}}\right)^{\frac{3}{2}}+a^{2} \leq a^{2}+c^{2}+|b|^{2}+2 a c,
$$

that is,

$$
\frac{c^{2}+|b|^{2}+2 a c}{\sqrt{2}\left(c^{\frac{4}{3}}+|b|^{\frac{4}{3}}\right)^{\frac{3}{2}}} \geq 1 .
$$

Divide both, numerator and denominator, by $a^{2}$ in order to convert the problem in having to achieve

$$
\frac{x^{2}+y^{2}+2 x}{\sqrt{2}\left(x^{\frac{4}{3}}+y^{\frac{4}{3}}\right)^{\frac{3}{2}}} \geq 1
$$

for $0 \leq y \leq x \leq 1$

(2) Assume next $a \geq|b| \geq c$. In this seciond part of the proof we shall need to employ a couple of real valued functions that will come in handy to achieve our purpose. Let us first focus our attention on the choice of the constants $a^{\prime}, b^{\prime}, c^{\prime}$, as before,

$$
a^{\prime}=\frac{\left(|c|^{\frac{4}{3}}+|b|^{\frac{4}{3}}\right)^{\frac{3}{4}}}{2^{\frac{3}{4}}}, c^{\prime}=-a^{\prime}, b^{\prime}=a .
$$

In this case, choose

$$
\lambda=\operatorname{sign}(\operatorname{Re}(b)) \sqrt{\frac{1}{2}}+i \operatorname{sign}(\operatorname{Im}(b)) \sqrt{\frac{1}{2}} .
$$

Then,

$$
\begin{aligned}
\|(a, b, c)\|_{\mathbb{D}}^{2} & \geq a^{2}+c^{2}+|b|^{2}+2\left[\sqrt{\frac{1}{2}}(a+c)|\operatorname{Re}(b)|+\sqrt{\frac{1}{2}}(a-c)|\operatorname{Im}(b)|\right] \\
& \geq a^{2}+c^{2}+|b|^{2}+\sqrt{2}|b|(a-c) .
\end{aligned}
$$

Hence, we will achieve the desired result if we can guarantee

$$
\sqrt{2}\left(|c|^{\frac{4}{3}}+|b|^{\frac{4}{3}}\right)^{\frac{3}{2}}+a^{2} \leq a^{2}+c^{2}+|b|^{2}+\sqrt{2}(a-c)|b|,
$$

in other words,

$$
1 \leq \Phi_{1}(x, y):=\frac{x^{2}+y^{2}+\sqrt{2}(1-x) y}{\sqrt{2}\left(x^{\frac{4}{3}}+y^{\frac{4}{3}}\right)^{\frac{3}{2}}},
$$

where $0 \leq x \leq y \leq 1$.

Let us focus now in another choice of constants $a^{\prime}, b^{\prime}, c^{\prime}$ :

$$
a^{\prime}=\frac{\left(|a|^{\frac{4}{3}}+|c|^{\frac{4}{3}}+|b|^{\frac{4}{3}}\right)^{\frac{3}{4}}}{\left(2+k^{4 / 3}\right)^{\frac{3}{4}}}, c^{\prime}=-a^{\prime}, b^{\prime}=k a,
$$


where $k$ has been chosen so that $\|(a, b, c)\|_{\frac{4}{3}}=\left\|\left(a^{\prime}, b^{\prime}, c^{\prime}\right)\right\|_{\frac{4}{3}}$. It can be proved that $k \approx 2.828$. Still giving the value $\lambda=\operatorname{sign}(\operatorname{Re}(b))$

$$
\|(a, b, c)\|_{\mathbb{D}}^{2} \geq a^{2}+c^{2}+|b|^{2}+2 a c,
$$

and again we guarantee that we achieve what we are searching for if we get

$$
4 a^{\prime 2}+b^{\prime 2}=\frac{\left(|a|^{\frac{4}{3}}+|c|^{\frac{4}{3}}+|b|^{\frac{4}{3}}\right)^{\frac{3}{2}}}{\left(2+k^{4 / 3}\right)^{\frac{3}{2}}}\left(4+k^{2}\right) \leq a^{2}+c^{2}+|b|^{2}+2 a c,
$$

in other words,

$$
1 \leq \Psi_{1}(x, y):=\frac{\left(1+x^{2}+y^{2}+2 y x\right)\left(2+k^{4 / 3}\right)^{\frac{3}{2}}}{\left(4+k^{2}\right)\left(y^{\frac{4}{3}}+x^{\frac{4}{3}}+1\right)^{\frac{3}{2}}},
$$

with $0 \leq x \leq y \leq 1$.

The reader can check using elementary calculus that, if

$$
H(x, y):=\max \left\{\Phi_{1}(x, y), \Psi_{1}(x, y)\right\}
$$

then

$$
1 \leq H(x, y) \text { for every } \quad 0 \leq x \leq y \leq 1
$$

(3) Assume finally $|b| \geq a \geq c$. Then, we may choose

$$
a^{\prime}=\frac{\left(|a|^{\frac{4}{3}}+|c|^{\frac{4}{3}}+|b|^{\frac{4}{3}}\right)^{\frac{3}{4}}}{\left(2+k^{4 / 3}\right)^{\frac{3}{4}}}, c^{\prime}=-a^{\prime}, b^{\prime}=k a,
$$

where $k$ is chosen as in the previous case. For $\lambda=\operatorname{sign}(\operatorname{Re}(b))$, we still need to make sure that

$$
1 \leq \Phi_{2}(x, y):=\frac{\left(1+x^{2}+y^{2}+2 x y\right)\left(2+k^{4 / 3}\right)^{\frac{3}{2}}}{\left(4+k^{2}\right)\left(x^{4 / 3}+y^{4 / 3}+1\right)^{\frac{3}{2}}} .
$$

For $\lambda=\operatorname{sign}(\operatorname{Re}(b)) \sqrt{\frac{1}{2}}+i \operatorname{sign}(\operatorname{Im}(b)) \sqrt{\frac{1}{2}}$, we need to make sure that

$$
1 \leq \Psi_{2}(x, y):=\frac{\left(1+x^{2}+y^{2}+\sqrt{2}(y-x)\left(2+k^{4 / 3}\right)^{\frac{3}{2}}\right.}{\left(4+k^{2}\right)\left(x^{4 / 3}+y^{4 / 3}+1\right)^{\frac{3}{2}}} .
$$

Next, choose

$$
a^{\prime}=\frac{\left(|a|^{4 / 3}+|c|^{4 / 3}\right)^{3 / 4}}{2^{3 / 4}}, \quad c^{\prime}=-a^{\prime} \quad \text { and } \quad b^{\prime}=|b|,
$$

such that

$$
\left\|\left(a^{\prime}, b^{\prime}, c^{\prime}\right)\right\|_{\mathbb{D}}^{2}=4 \frac{\left(|a|^{4 / 3}+|c|^{4 / 3}\right)^{3 / 2}}{2^{3 / 2}}+|b|^{2}
$$

and

$$
\lambda=\operatorname{sign}(\operatorname{Re}(b)) \sqrt{1-\left(\frac{a-c}{|b|}\right)^{2}}+i \operatorname{sign}(\operatorname{Im}(b)) \frac{a-c}{|b|} .
$$


In that case,

$$
\begin{aligned}
\|(a, b, c)\|_{\mathbb{D}}^{2} \geq & a^{2}+c^{2}+|b|^{2}+2\left[a c \operatorname{Re}\left(\lambda^{2}\right)+(a+c) \operatorname{Re}(b) \operatorname{Re}(\lambda)+(a-c) \operatorname{Im}(b) \operatorname{Im}(\lambda)\right] \\
= & a^{2}+c^{2}+|b|^{2}+2\left[a c\left(1-2\left(\frac{a-c}{|b|}\right)^{2}\right)+(a+c)|\operatorname{Re}(b)| \sqrt{1-\left(\frac{a-c}{|b|}\right)^{2}}\right. \\
& \left.+(a-c)|\operatorname{Im}(b)| \frac{a-c}{|b|}\right] .
\end{aligned}
$$

Assume first $|\operatorname{Im}(b)| \geq \frac{\sqrt{2}}{2}$. Then,

$$
\begin{aligned}
\|(a, b, c)\|_{\mathbb{D}}^{2} & \geq a^{2}+c^{2}+|b|^{2} \\
& +2\left[a c\left(1-2\left(\frac{a-c}{|b|}\right)^{2}\right)+(a-c) \frac{\sqrt{2}}{2}|b| \frac{a-c}{|b|}\right] .
\end{aligned}
$$

Hence, we will achieve what we are searching for if we can assure that

$$
1 \leq \Omega_{2}^{(1)}(x, y):=\frac{x^{2}+y^{2}+2 x y\left(1-2(y-x)^{2}\right)+\sqrt{2}(y-x)^{2}}{\sqrt{2}\left(x^{4 / 3}+y^{4 / 3}\right)^{\frac{3}{2}}} .
$$

On in on, we need to prove

$$
1 \leq \max \left\{\Phi_{2}(x, y), \Psi_{2}(x, y), \Omega_{2}^{(1)}(x, y)\right\},
$$

for $0 \leq x \leq y \leq 1$. This can be done by means of elementary calculus, and we leave it as an exercise to the reader.

On the other hand if, instead, we have $|\operatorname{Re}(b)| \geq \frac{\sqrt{2}}{2}$, then

$$
\begin{aligned}
\|(a, b, c)\|_{\mathbb{D}}^{2} & \geq a^{2}+c^{2}+|b|^{2} \\
& +2\left[a c\left(1-2\left(\frac{a-c}{|b|}\right)^{2}\right)+(a+c) \frac{\sqrt{2}}{2}|b| \sqrt{1-\left(\frac{a-c}{|b|}\right)^{2}}\right],
\end{aligned}
$$

and (in this case) we will be working with the condition

$$
1 \leq \Omega_{2}^{(2)}(x, y):=\frac{x^{2}+y^{2}+2 x y\left(1-2(y-x)^{2}\right)+\sqrt{2}(y+x) \sqrt{1-(y-x)^{2}}}{\sqrt{2}\left(x^{4 / 3}+y^{4 / 3}\right)^{\frac{3}{2}}},
$$

and, in conclusion, we shall need to guarantee that

$$
1 \leq \max \left\{\Phi_{2}(x, y), \Psi_{2}(x, y), \Omega_{2}^{(2)}(x, y)\right\},
$$

for $0 \leq x \leq y \leq 1$, which we also leave as an exercise to the reader.

And, with this last case, the proof is complete.

In order to prove that $D_{\mathbb{C}, 2}(2)=\sqrt[4]{\frac{3}{2}}$ we will also need the following description of the extreme points of the unit ball of $\mathbb{R}^{3}$ endowed with the norm

$$
\|(a, b, c)\|_{\mathbb{D}}:=\sup \left\{\left|a z^{2}+b z+c\right|:|z| \leq 1\right\}
$$

for $a, b, c \in \mathbb{R}$. This norm has been studied by Aron and klimek in [1], where they denote it by $\|\cdot\|_{\mathbb{C}}$. Observe, once again that

$$
\|(a, b, c)\|_{\mathbb{D}}=\left\|a z^{2}+b w z+c z^{2}\right\|_{\mathbb{D}} .
$$


Theorem 2.2 (Aron and Klimek, [1]). Let $E_{\mathbb{R}}$ be the real subspace of $\mathcal{P}\left({ }^{2} \ell_{\infty}^{2}(\mathbb{C})\right.$ ) given by $\left\{a z^{2}+b w z+c w^{2}:(a, b, c) \in \mathbb{R}^{3}\right\}$. Then

$$
\operatorname{ext}\left(\mathrm{B}_{E_{\mathbb{R}}}\right)=\left\{\left(s, \sqrt{4|s||t|\left(\frac{1}{(|s|+|t|)^{2}}-1\right)}, t\right):(s, t) \in G\right\}
$$

where $\operatorname{ext}\left(\mathrm{B}_{E_{\mathbb{R}}}\right)$ is the set of extreme points of the unit ball of $E_{\mathbb{R}}$, namely $\mathrm{B}_{E_{\mathbb{R}}}$ and $G=$ $\left\{(s, t) \in \mathbb{R}^{2}:|s|+|t|<1\right.$ and $\left.|s+t| \leq(s+t)^{2}\right\} \cup\{ \pm(1,0), \pm(0,1)\}$.

Theorem 2.3. The optimal complex polynomial Bohnenblust-Hille constant for polynomials in $E_{\mathbb{R}}$, which we denote by $D_{\mathbb{C}, 2}\left(E_{\mathbb{R}}\right)$, is given by $D_{\mathbb{C}, 2}\left(E_{\mathbb{R}}\right)=\sqrt[4]{\frac{3}{2}}$. Moreover,

$$
D_{\mathbb{C}, 2}(2)=\sqrt[4]{\frac{3}{2}} \approx 1.1066
$$

Proof. Using convexity we have

$$
\begin{aligned}
D_{\mathbb{C}, 2}\left(E_{\mathbb{R}}\right) & =\sup \left\{\|(a, b, c)\|_{\frac{4}{3}}:\left\|a z_{1}^{2}+b z_{1} z_{2}+c z_{2}^{2}\right\| \leq 1\right\} \\
& =\sup \left\{\|(a, b, c)\|_{\frac{4}{3}}:\|(a, b, c)\|_{\mathbb{C}} \leq 1\right\} \\
& =\sup \left\{\|(a, b, c)\|_{\frac{4}{3}}:(a, b, c) \in \operatorname{ext}\left(\mathrm{B}_{E_{\mathbb{R}}}\right)\right\},
\end{aligned}
$$

Hence

$$
D_{\mathbb{C}, 2}\left(E_{\mathbb{R}}\right)=\sup \left\{\left(|s|^{\frac{4}{3}}+|t|^{\frac{4}{3}}+\left[4|s||t|\left(\frac{1}{(|s|+|t|)^{2}}-1\right)\right]^{\frac{2}{3}}\right)^{\frac{3}{4}}:(s, t) \in G\right\} .
$$

If $\Phi(s, t)=\left(|s|^{\frac{4}{3}}+|t|^{\frac{4}{3}}+\left[4|s||t|\left(\frac{1}{(|s|+|t|)^{2}}-1\right)\right]^{\frac{2}{3}}\right)^{\frac{3}{4}}$ for $(s, t) \in G$, one can prove using elementary calculus that $\Phi$ attains its maximum on $G$ at $\pm\left(\frac{\sqrt{3}}{6},-\frac{\sqrt{3}}{6}\right)$ and $\Phi\left(\frac{\sqrt{3}}{6},-\frac{\sqrt{3}}{6}\right)=$ $\sqrt[4]{\frac{3}{2}}$. Finally, from Lemma 2.1 we also obtain that $D_{\mathbb{C}, 2}(2)=\sqrt[4]{\frac{3}{2}} \approx 1.1066$.

Observe that the fact that $D_{\mathbb{C}, 2}(2) \geq \sqrt[4]{\frac{3}{2}}$ was already proved in [22]. The reader can find a sketch of the graph of $\Phi$ on the part of $G$ contained in the second quadrant in Figure 1.

\section{The EXACT VAlue of $D_{\mathbb{R}, 2}(2)$ AND LOWER BOUndS FOR $D_{\mathbb{R}, m}(2)$}

In [7] it is proved that the asymptotic hypercontractivity constant of the real polynomial $\mathrm{BH}$ inequality is exactly 2 . Is it true that $H_{\mathbb{R}, \infty}(2)=2$ ? The results presented here suggest that, perhaps $H_{\mathbb{R}, \infty}(2)<2$. In this section, as we did in the previous one, we will also identify polynomials with the vector of its coefficients.

Remark 3.1. Throughout this section we will compute several times norms of polynomials on the real line numerically. This is done by using Matlab. In particular, if $P(x)$ is a real polynomial on $\mathbb{R}$, we apply the predefined Matlab function roots.m to $P^{\prime}$ in order to obtain an approximation of all the critical points of $P$. If $x_{1}, \ldots, x_{k}$ are all the roots of $P^{\prime}$ in $[-1,1]$, then we approach the norm of $P$ as

$$
\|P\|:=\max \{|P(x)|: x \in[-1,1]\}=\max \left\{\left|P\left(x_{i}\right)\right|,|P( \pm 1)|: i=1, \ldots, k\right\} .
$$

Another Matlab predefined function, namely conv.m, is used in order to to multiply polynomials. This is done to obtain Figure 4. 


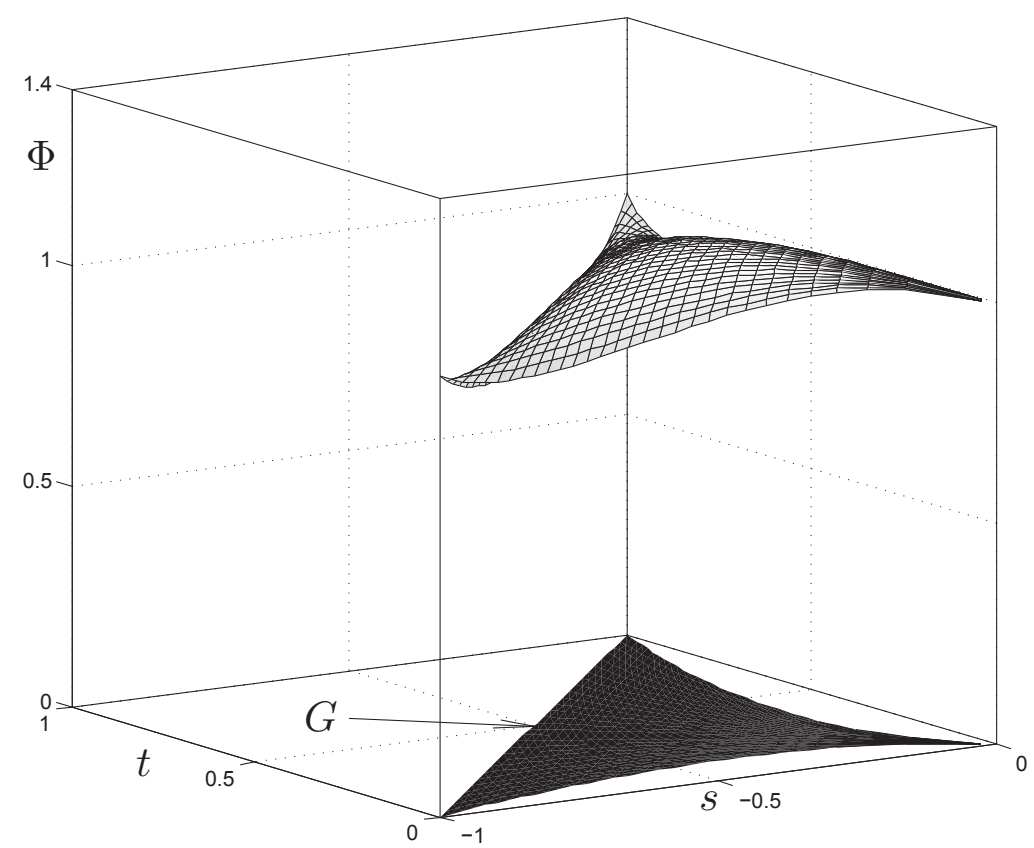

FiguRE 1. Graph of $\Phi$ on $G$ intersected with the second quadrant.

3.1. The exact calculation of $D_{\mathbb{R}, 2}(2)$. The value of the constant $D_{\mathbb{R}, 2}(2)$ can be obtained using the geometry of the unit ball of $\mathcal{P}\left(\ell_{\infty}^{2}(\mathbb{R})\right)$ described in [8]. We state the result we need for completeness:

Theorem 3.2. [Choi, Kim [8]] The set $\operatorname{ext}\left(\mathrm{B}_{\mathcal{P}\left({ }^{2} \ell_{\infty}^{2}(\mathbb{R})\right)}\right)$ of extreme points of the unit ball of $\mathcal{P}\left({ }^{2} \ell_{\infty}^{2}(\mathbb{R})\right)$ is given by

$$
\operatorname{ext}\left(\mathrm{B}_{\mathcal{P}\left({ }^{2} \ell_{\infty}^{2}(\mathbb{R})\right)}\right)=\left\{ \pm x^{2}, \pm y^{2}, \pm\left(t x^{2}-t y^{2} \pm 2 \sqrt{t(1-t)} x y\right): t \in[1 / 2,1]\right\} .
$$

As a consequence of the previous result, we obtain the following:

Theorem 3.3. Let $f$ be the real valued function given by

$$
f(t)=\left[2 t^{\frac{4}{3}}+(2 \sqrt{t(1-t)})^{\frac{4}{3}}\right]^{\frac{3}{4}} .
$$

We have that $D_{\mathbb{R}, 2}(2)=f\left(t_{0}\right) \approx 1.837373$, where

$$
t_{0}=\frac{1}{36}(2 \sqrt[3]{107+9 \sqrt{129}}+\sqrt[3]{856-72 \sqrt{129}}+16) \approx 0.867835 .
$$

The exact value of $f\left(t_{0}\right)$ is given by

$$
\left(\frac{(2 \sqrt[3]{107+9 \sqrt{129}}+\sqrt[3]{856-72 \sqrt{129}}+16)^{4 / 3}}{186^{2 / 3}}+\frac{1}{9\left(-\frac{3}{-2 \sqrt[3]{107+9 \sqrt{129}}+(107+9 \sqrt{129})^{2 / 3}-2 \sqrt[3]{107-9 \sqrt{129}}+(107-9 \sqrt{129})^{2 / 3}-60}\right)^{2 / 3}}\right)^{3 / 4},
$$

Moreover, the following normalized polynomials are extreme for this problem:

$$
P_{2}(x, y)= \pm\left(t_{0} x^{2}-t_{0} y^{2} \pm 2 \sqrt{t_{0}\left(1-t_{0}\right)} x y\right) .
$$


Proof. Let

$$
f(t)=\left[2 t^{\frac{4}{3}}+(2 \sqrt{t(1-t)})^{\frac{4}{3}}\right]^{\frac{3}{4}} .
$$

We just have to notice that due to the convexity of the $\ell_{p}$-norms and Theorem 3.2 we have

$$
\begin{aligned}
D_{\mathbb{R}, 2}(2) & =\sup \left\{|\mathbf{a}|_{\frac{4}{3}}: \mathbf{a} \in \mathrm{B}_{\mathcal{P}\left({ }^{2} \ell_{\infty}^{2} \mathbb{R}\right)}\right\} \\
& =\sup \left\{|\mathbf{a}|_{\frac{4}{3}}: \mathbf{a} \in \operatorname{ext}\left(\mathrm{B}_{\mathcal{P}\left({ }^{2} \ell_{\infty}^{2} \mathbb{R}\right)}\right)\right\}=\sup _{t \in[1 / 2,1]} f(t) .
\end{aligned}
$$

Some calculations will show that the last supremum is attained at $t=t_{0}$, concluding the proof.

Now, if $\mathbf{a}_{n}$ is the vector of the coefficients of $P_{2}^{n}$ for each $n \in \mathbb{N}$, then we know that

$$
D_{\mathbb{R}, 2 n}(2) \geq \frac{\left|\mathbf{a}_{n}\right| \frac{4 n}{2 n+1}}{\left\|P_{2}\right\|^{n}} .
$$

Since $\left\|P_{2}\right\|=1$, then (3.1) with $n=300$ (see also Figure 4 ) proves that

$$
D_{\mathbb{R}, 600}(2) \geq(1.36117)^{600},
$$

providing numerical evidence showing that

$$
H_{\mathbb{R}, \infty}(2) \geq 1.36117 \text {. }
$$

3.2. Educated guess for the exact calculation of $D_{\mathbb{R}, 3}(2)$. To the authors' knowledge the calculation of $\|P\|$ is, in general, far from being easy. However there is a way to compute $\|P\|$ for specific cases. For instance Grecu, Muñoz and Seoane prove in [18, Lemma 3.12] the following formula:

Lemma 3.4. If for every $a, b \in \mathbb{R}$ we define $P_{a, b}(x, y)=a x^{3}+b x^{2} y+b x y^{2}+a y^{3}$ then

$$
\left\|P_{a, b}\right\|= \begin{cases}\left|a-\frac{b^{2}}{3 a}+\frac{2 b^{3}}{27 a^{2}}+\frac{2 a}{27}\left(-\frac{3 b}{a}+\frac{b^{2}}{a^{2}}\right)^{\frac{3}{2}}\right| & \text { if } a \neq 0 \text { and } b_{1}<\frac{b}{a}<3-2 \sqrt{3} \\ |2 a+2 b| & \text { otherwise }\end{cases}
$$

where

$$
b_{1}=\frac{3}{7}\left(3-\frac{2 \sqrt[3]{9}}{\sqrt[3]{-12+7 \sqrt{3}}}+2 \sqrt[3]{-36+21 \sqrt{3}}\right) \approx-1.6692 .
$$

From Lemma 3.4 we have the following sharp polynomial Bohnenblust-Hille type constant:

Theorem 3.5. Let $P_{a, b}(x, y)=a x^{3}+b x^{2} y+b x y^{2}+a y^{3}$ for $a, b \in \mathbb{R}$ and consider the subset of $\mathcal{P}\left({ }^{3} \ell_{\infty}^{2}(\mathbb{R})\right)$ given by $E=\left\{P_{a, b}: a, b \in \mathbb{R}\right\}$. Then

$$
\frac{|(a, b, b, a)|_{\frac{3}{2}}}{\left\|P_{a, b}\right\|}=\left\{\begin{array}{c}
\frac{27 a^{2}\left(2|a|^{\frac{3}{2}}+2|b|^{\frac{3}{2}}\right)^{\frac{2}{3}}}{\left|27 a^{3}-9 a b^{2}+2 b^{3}+2 \operatorname{sign}(a)\left(-3 a b+b^{2}\right)^{\frac{3}{2}}\right|^{3}}, \text { if } a \neq 0 \text { and } b_{1}<\frac{b}{a}<3-2 \sqrt{3}, \\
\frac{\left(2|a|^{\frac{3}{2}}+2|b|^{\frac{3}{2}}\right)^{\frac{2}{3}}}{2|a+b|}, \text { otherwise }
\end{array}\right.
$$

where $b_{1}$ was defined in Lemma 3.4. Moreover, the above function attains its maximum when $\frac{b}{a}=b_{1}$, which implies that

$$
D_{\mathbb{R}, 3}(E)=\frac{\left(2+2\left|b_{1}\right|^{\frac{3}{2}}\right)^{\frac{2}{3}}}{2\left|1+b_{1}\right|} \approx 2.5525
$$




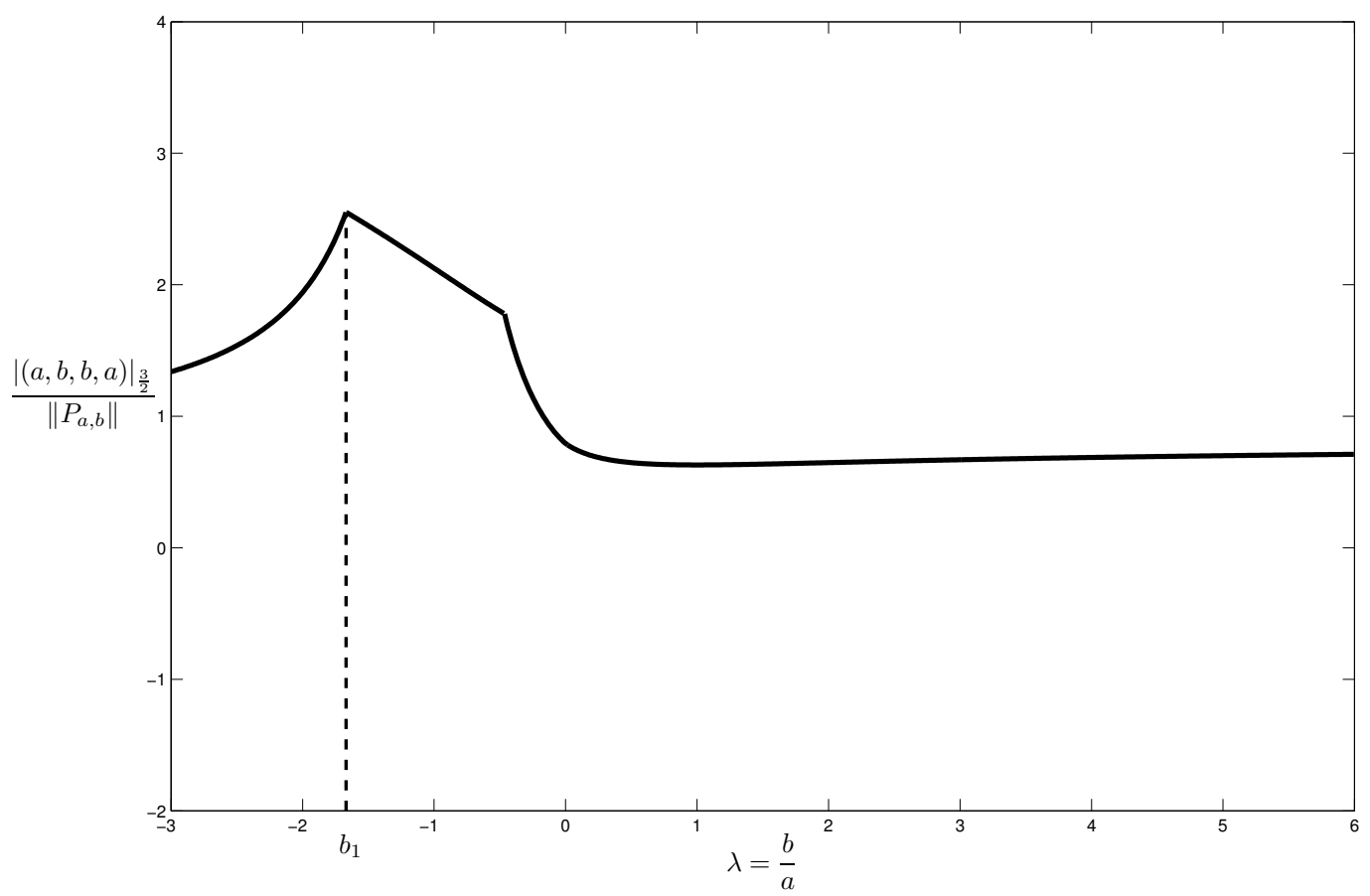

FIGURE 2. Graph of the quotient $\frac{|(a, b, b, a)|_{\frac{3}{2}}}{\left\|P_{a, b}\right\|}$ as a function of $\lambda=\frac{a}{b}$.

The authors have numerical evidence to state that

$$
D_{\mathbb{R}, 3}(2)=D_{\mathbb{R}, 3}(E) .
$$

Moreover, one polynomial for which $D_{\mathbb{R}, 3}(2)$ would be attained is

$$
P_{3}(x, y)=x^{3}+b_{1} x^{2} y+b_{1} x y^{2}+y^{3},
$$

where $b_{1} \approx-1.6692$ is as in Lemma 3.4. It can be proved from Lemma 3.4 that

$$
\left\|P_{3}\right\| \approx 1.33848
$$

up to 5 decimal places. If $\mathbf{a}_{\mathbf{n}}$ is the vector of the coefficients of $P_{3}(x, y)^{n}$ and we use the fact that

$$
D_{\mathbb{R}, 3 n}(2) \geq \frac{\left|\mathbf{a}_{n}\right|_{\frac{6 n}{3 n+1}}}{\left\|P_{3}\right\|^{n}},
$$

then putting $n=200$ in (3.2) we obtain, for instance,

$$
D_{\mathbb{R}, 600}(2) \geq(1.42234)^{600},
$$

which provides numerical evidence showing that

$$
H_{\mathbb{R}, \infty}(2) \geq 1.42234 \text {. }
$$

3.3. Numerical calculation of $D_{\mathbb{R}, 5}(2)$. Let us define the polynomial

$$
P_{5}(x, y)=a x^{5}-b x^{4} y-c x^{3} y^{2}+c x^{2} y^{3}+b x y^{4}-a y^{5},
$$


with

$$
\begin{aligned}
& a=0.19462, \\
& b=0.66008, \\
& c=0.97833 .
\end{aligned}
$$

The norm of $P_{5}$ can be calculated numerically (using Remark 3.1), and it turns out to be

$$
\left\|P_{5}\right\|=0.28617
$$

up to 5 decimal places. The authors have numerical evidence showing that

$$
D_{\mathbb{R}, 5}(2) \approx 6.83591 \text {. }
$$

In any case we have

$$
D_{\mathbb{R}, 5}(2) \geq \frac{|(a,-b,-c, c, b,-a)|_{\frac{5}{3}}}{\left\|P_{5}\right\|} \approx 6.83591 .
$$

It is interesting to observe that we can improve numerically the estimate $H_{\infty, \mathbb{R}}(2) \geq \sqrt[8]{27} \approx$ 1.50980 (see [7, Theorem 4.2]) by considering polynomials of the form $P_{5}^{n}$. Indeed, if $\mathbf{a}_{n}$ is the vector of the coefficients of $P_{5}^{n}$ for each $n \in \mathbb{N}$, then we know that

$$
D_{\mathbb{R}, 5 n}(2) \geq \frac{\left|\mathbf{a}_{n}\right| \frac{10 n}{5 n+1}}{\left\|P_{5}\right\|^{n}},
$$

Using (3.3) with $n=120$ we obtain, in particular (see also Figure 4)

$$
D_{\mathbb{R}, 600}(2) \geq(1.54987)^{600},
$$

providing numerical evidence showing that

$$
H_{\mathbb{R}, \infty}(2) \geq 1.54987 \text {. }
$$

3.4. Educated guess for the exact calculation of $D_{\mathbb{R}, 6}(2)$. The authors have numerical evidence pointing to the fact that an extreme polynomial in the Bohnenblust-Hille inequality for polynomials in $\mathcal{P}\left({ }^{6} \ell_{\infty}^{2}(\mathbb{R})\right)$ may be of the form

$$
Q_{a, b}(x, y)=a x^{5} y+b x^{3} y^{3}+a x y^{5} .
$$

This motivates a deeper study of this type of polynomials, which we do in the following result.

Theorem 3.6. Let $Q_{a, b}(x, y)=a x^{5} y+b x^{3} y^{3}+a x y^{5}$ for $a, b \in \mathbb{R}$ and consider the subspace of $\mathcal{P}\left({ }^{6} \ell_{\infty}^{2}(\mathbb{R})\right)$ given by $F=\left\{Q_{a, b}: a, b \in \mathbb{R}\right\}$. Suppose $\lambda_{0}<\lambda_{1}$ are the only two roots of the equation

$$
\frac{\left|3 \lambda^{2}-20+\lambda \sqrt{9 \lambda^{2}-20}\right|}{25} \sqrt{\frac{-3 \lambda-\sqrt{9 \lambda^{2}-20}}{10}}=|2+\lambda| .
$$

Then if $\lambda=\frac{b}{a}$ we have

$$
\frac{|(0, a, 0, b, 0, a, 0)|_{\frac{12}{7}}}{\left\|Q_{a, b}\right\|}=\left\{\begin{array}{c}
\frac{25 \sqrt{10}\left(2+|\lambda|^{\frac{12}{7}}\right)^{\frac{12}{7}}}{\left|3 \lambda^{2}-20+\lambda \sqrt{9 \lambda^{2}-20}\right| \sqrt{-3 \lambda-\sqrt{9 \lambda^{2}-20}},} \text { if } a \neq 0 \text { and } \lambda_{0}<\frac{b}{a}<\lambda_{1} \\
\frac{\left(2+|\lambda|^{\frac{12}{7}}\right)^{\frac{7}{12}}}{|2+\lambda|}, \text { otherwise. }
\end{array}\right.
$$


Observe that $\lambda_{0} \approx-2.2654, \lambda_{1} \approx-1.6779$ and the above function attains its maximum when $\frac{b}{a}=\lambda_{0}$ (see Figure 3), which implies that

$$
D_{\mathbb{R}, 6}(F)=\frac{\left(2+\left|\lambda_{0}\right|^{\frac{12}{7}}\right)^{\frac{12}{7}}}{\left|2+\lambda_{0}\right|} \approx 10.7809 .
$$

Proof. We do not lose generality by considering only polynomials of the form $Q_{1, \lambda}$, in which case

$$
\left\|Q_{1, \lambda}\right\|=\sup \left\{\left|x^{5}+\lambda x^{3}+x\right|: x \in[0,1]\right\} .
$$

The polynomial $q_{\lambda}(x):=x^{5}+\lambda x^{3}+x$ has no critical points if $\lambda>-\frac{2 \sqrt{5}}{3}$, otherwise it has the following critical points in $[0,1]$ :

$$
x_{0}:=\sqrt{\frac{-3 \lambda-\sqrt{9 \lambda^{2}-20}}{10}} \text { and } x_{1}:=\sqrt{\frac{-3 \lambda+\sqrt{9 \lambda^{2}-20}}{10}} \text { if }-2 \leq \lambda \leq-\frac{2 \sqrt{5}}{3},
$$

and $x_{0}$ if $\lambda \leq-2$. Notice that

$$
\begin{aligned}
& q_{\lambda}\left(x_{0}\right)=\frac{-3 \lambda^{2}+20-\lambda \sqrt{9 \lambda^{2}-20}}{20} x_{0} \\
& q_{\lambda}\left(x_{1}\right)=\frac{-3 \lambda^{2}+20+\lambda \sqrt{9 \lambda^{2}-20}}{20} x_{1} .
\end{aligned}
$$

It is easy to check that $\left|q_{\lambda}\left(x_{0}\right)\right| \geq\left|q_{\lambda}\left(x_{1}\right)\right|$ for $-2 \leq \lambda \leq-\frac{2 \sqrt{5}}{3}$, which implies that

$$
\left\|Q_{1, \lambda}\right\|= \begin{cases}\max \left\{|2+\lambda|,\left|q_{\lambda}\left(x_{0}\right)\right|\right\} & \text { if }-2 \leq \lambda \leq-\frac{2 \sqrt{5}}{3} \\ |2+\lambda| & \text { otherwise. }\end{cases}
$$

The equation $|2+\lambda|=\left|q_{\lambda}\left(x_{0}\right)\right|$ turns out to have only two roots, namely $\lambda_{0} \approx-2.2654$ and $\lambda_{1} \approx-1.6779$. By continuity, it is easy to prove that $|2+\lambda| \leq\left|q_{\lambda}\left(x_{0}\right)\right|$ only if $-2 \leq \lambda \leq-\frac{2 \sqrt{5}}{3}$, which concludes the proof.

As mentioned above, we have numerical evidence showing that

$$
D_{\mathbb{R}, 6}(2)=D_{\mathbb{R}, 6}(F)=\frac{\left(2+\left|\lambda_{0}\right|^{\frac{12}{7}}\right)^{\frac{12}{7}}}{\left|2+\lambda_{0}\right|} \approx 10.7809 .
$$

In any case we do have that

$$
D_{\mathbb{R}, 6}(2) \geq 10.7809 .
$$

As we did in the previous cases, it would be interesting to know if we can improve numerically our best lower bound on $H_{\mathbb{R}, \infty}$ by considering powers of

$$
P_{6}(x, y)=Q_{1, \lambda_{0}}(x, y)=x^{5} y+\lambda_{0} x^{3} y^{3}+x y^{5},
$$

with $\lambda_{0}$ as in Theorem $3.6\left(\lambda_{0} \approx-2.2654\right)$. If $\mathbf{a}_{n}$ is the vector of the coefficients of $P_{6}^{n}$ for each $n \in \mathbb{N}$, then we know that

$$
D_{\mathbb{R}, 6 n}(2) \geq \frac{\left|\mathbf{a}_{n}\right|_{\frac{12 n}{6 n+1}}}{\left\|P_{6}\right\|^{n}} .
$$

Using (3.4) with $n=100$ and estimating $\left\|P_{6}\right\|$ according to Remark 3.1 we obtain

$$
D_{\mathbb{R}, 600}(2) \geq(1.58432)^{600},
$$




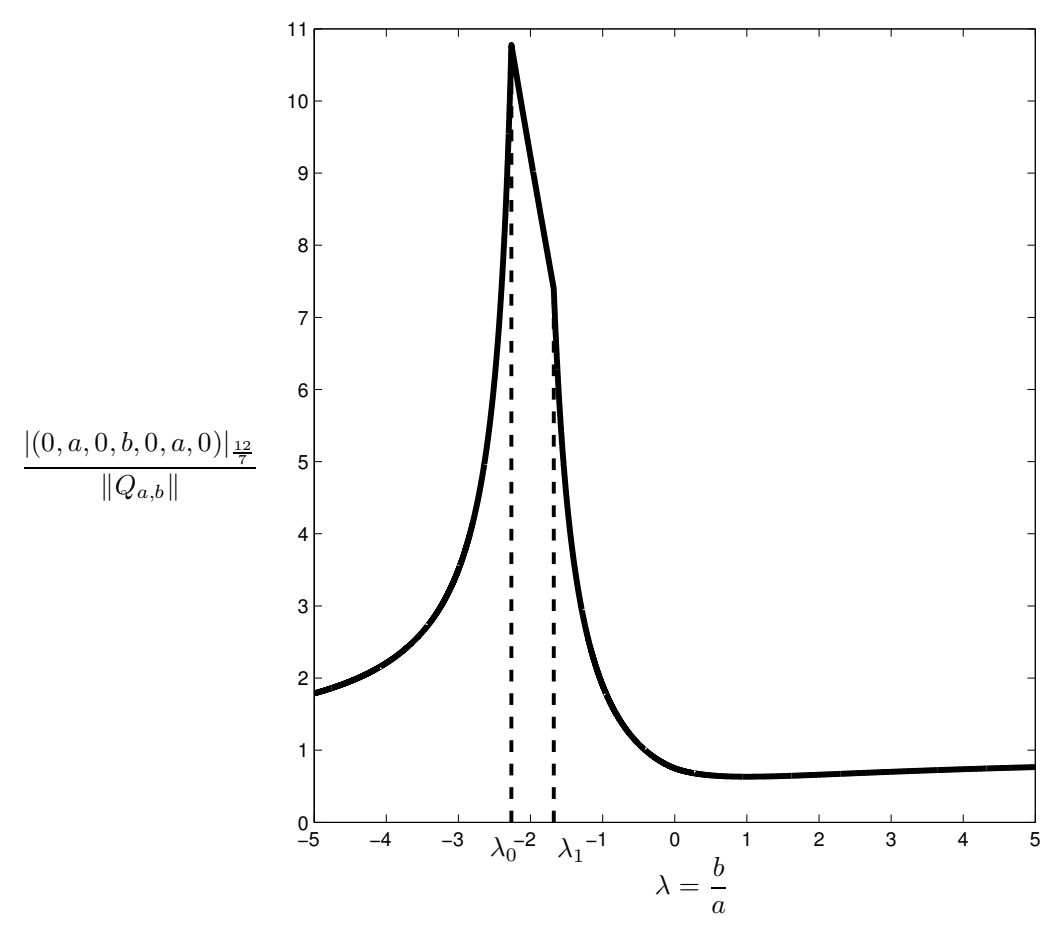

FIGURE 3. Graph of the quotient $\frac{|(0, a, 0, b, 0, a, 0)|_{\frac{12}{7}}}{\left\|Q_{a, b}\right\|}$ as a function of $\lambda=\frac{b}{a}$.

which suggests that (see Figure 4)

$$
H_{\infty, \mathbb{R}}(2) \geq 1.58432 .
$$

3.5. Numerical calculation of $D_{\mathbb{R}, 7}(2)$. Let us define the polynomial

$$
P_{7}(x, y)=-a x^{7}+b x^{6} y+c x^{5} y^{2}-d x^{4} y^{3}-d x^{3} y^{4}+c x^{2} y^{5}+b x y^{6}-a y^{7},
$$

with

$$
\begin{aligned}
a & =0.05126, \\
b & =0.22070, \\
c & =0.50537, \\
d & =0.71044 .
\end{aligned}
$$

It can be proved numerically (using Remark 3.1) that

$$
\left\|P_{7}\right\| \approx 0.07138
$$

up to 5 decimal places. The authors have numerical evidence showing that

$$
D_{\mathbb{R}, 7}(2) \approx \frac{|(-a, b, c,-d,-d, c, b,-a)|_{\frac{7}{4}}}{\left\|P_{7}\right\|} \approx 19.96308 .
$$

If $\mathbf{a}_{n}$ is the vector of the coefficients of $P_{7}^{n}$ for each $n \in \mathbb{N}$, then we know that

$$
D_{\mathbb{R}, 7 n}(2) \geq \frac{\left|\mathbf{a}_{n}\right|_{\frac{14 n}{7 n+1}}}{\left\|P_{7}\right\|^{n}} .
$$


Moreover, if we put $n=86$ in (3.5) we obtain

$$
D_{\mathbb{R}, 602}(2) \geq(1.61725)^{602},
$$

suggesting that

$$
H_{\mathbb{R}, \infty}(2) \geq 1.61725 .
$$

3.6. Numerical calculation of $D_{\mathbb{R}, 8}(2)$. Let us define the polynomial

$$
P_{8}(x, y)=-a x^{7} y+b x^{5} y^{3}-b x^{3} y^{5}+a x y^{7},
$$

with

$$
\begin{aligned}
a & =0.15258, \\
b & =0.64697 .
\end{aligned}
$$

It can be established numerically (see Remark 3.1) that

$$
\left\|P_{8}\right\| \approx 0.02985
$$

up to 5 decimal places. The authors have numerical evidence showing that

$$
D_{\mathbb{R}, 8}(2) \approx \frac{|(0,-a, 0, b, 0,-b, 0, a, 0)|_{\frac{16}{9}}}{\left\|P_{8}\right\|} \approx 33.36323 .
$$

If $\mathbf{a}_{n}$ is the vector of the coefficients of $P_{8}^{n}$ for each $n \in \mathbb{N}$, then we know that

$$
D_{\mathbb{R}, 8 n}(2) \geq \frac{\left|\mathbf{a}_{n}\right|_{\frac{16 n}{8 n+1}}}{\left\|P_{8}\right\|^{n}} .
$$

Moreover, using (3.6) with $n=75$ we obtain

$$
D_{\mathbb{R}, 600}(2) \geq(1.64042)^{600},
$$

which suggests that

$$
H_{\mathbb{R}, \infty}(2) \geq 1.64042 .
$$

3.7. Numerical calculation of $D_{\mathbb{R}, 10}(2)$. In this case our numerical estimates show that there exists an extreme polynomial in the Bohnenblust-Hille polynomial inequality in $\mathcal{P}\left({ }^{10} \ell_{\infty}^{2}(\mathbb{R})\right)$ of the form

$$
P_{10}(x, y)=a x^{9} y+b x^{7} y^{3}+x^{5} y^{5}+b x^{3} y^{7}+a x y^{9},
$$

with

$$
\begin{aligned}
& a=0.0938, \\
& b=-0.5938 .
\end{aligned}
$$

It can be computed numerically (see Remark 3.1) that

$$
\left\|P_{10}\right\| \approx 0.01530 \text {, }
$$

up to 5 decimal places. The authors have numerical evidence showing that

$$
D_{\mathbb{R}, 10}(2) \approx \frac{|(0, a, 0, b, 0,1,0, b, 0, a, 0)|_{\frac{20}{11}}}{\left\|P_{10}\right\|} \approx 90.35556 .
$$

If $\mathbf{a}_{n}$ is the vector of the coefficients of $P_{10}^{n}$ for each $n \in \mathbb{N}$, then we know that

$$
D_{\mathbb{R}, 10 n}(2) \geq \frac{\left|\mathbf{a}_{n}\right|_{\frac{20 n}{10 n+1}}}{\left\|P_{10}\right\|^{n}} .
$$




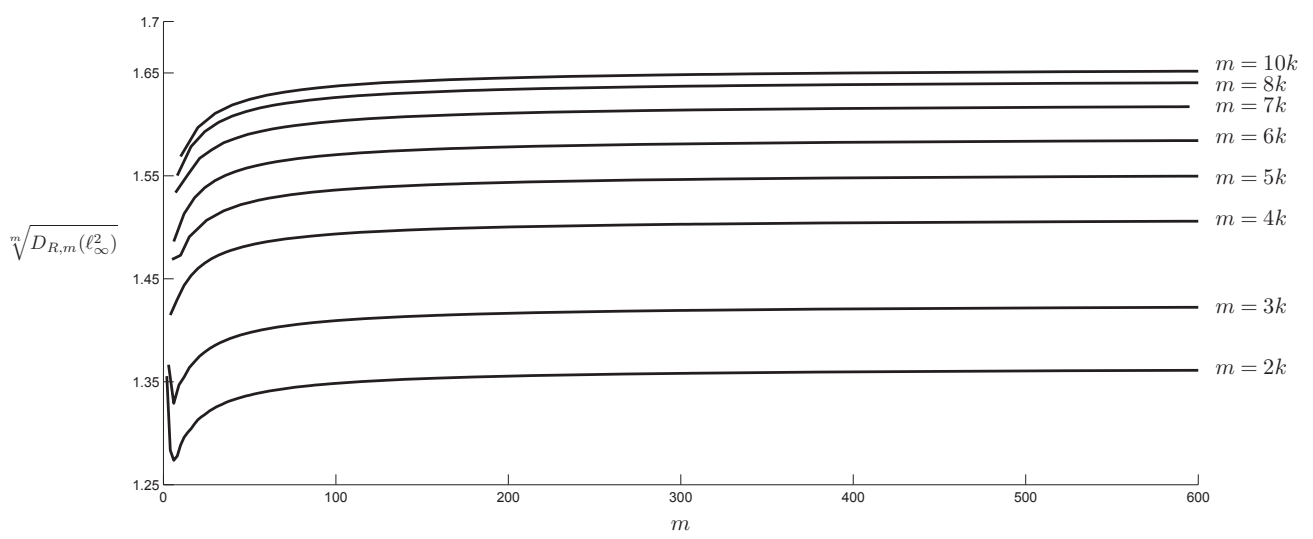

Figure 4. Graphs of the estimates on $\sqrt[m]{D_{\mathbb{R}, m}(2)}$ obtained by using (3.1) through (3.7).

If we set $n=60$ in (3.7) then we obtain

$$
D_{\mathbb{R}, 600}(2) \geq(1.65171)^{600},
$$

which suggests that

$$
H_{\mathbb{R}, \infty}(2) \geq 1.65171 .
$$

We have sketched in Figure 4 a summary of the numerical results obtained in this section.

\section{REFERENCES}

[1] R. M. Aron and M. Klimek, Supremum norms for quadratic polynomials, Arch. Math. (Basel) 76 (2001), no. $1,73-80$.

[2] F. Bayart, D. Pellegrino, and J. B. Seoane-Sepúlveda, The Bohr radius of the $n$-dimensional polydisk is equivalent to $\sqrt{(\log n) / n}$, Adv. Math. 264 (2014), 726-746.

[3] O. Blasco, The Bohr radius of a Banach space, Vector measures, integration and related topics, Oper. Theory Adv. Appl., vol. 201, Birkhäuser Verlag, Basel, 2010, pp. 59-64.

[4] H. P. Boas, The football player and the infinite series, Notices Amer. Math. Soc. 44 (1997), no. 11, 1430-1435.

[5] H. F. Bohnenblust and E. Hille, On the absolute convergence of Dirichlet series, Ann. of Math. (2) 32 (1931), no. 3, 600-622.

[6] E. Bombieri and J. Bourgain, A remark on Bohr's inequality, Int. Math. Res. Not. 80 (2004), 4307-4330.

[7] J. R. Campos, P. Jiménez-Rodríguez, G. A. Muñoz-Fernández, D. Pellegrino, and J. B. SeoaneSepúlveda, On the real polynomial Bohnenblust-Hille inequality, Linear Algebra Appl. 465 (2015), 391400.

[8] Y. S. Choi and S. G. Kim, The unit ball of $\mathcal{P}\left({ }^{2} l_{2}^{2}\right)$, Arch. Math. (Basel) 71 (1998), no. 6, $472-480$.

[9] A. Defant and L. Frerick, Hypercontractivity of the Bohnenblust-Hille inequality for polynomials and multidimensional Bohr radii, arXiv:0903.3395.

[10] A. Defant, L. Frerick, J. Ortega-Cerdà, M. Ounaïes, and K. Seip, The Bohnenblust-Hille inequality for homogeneous polynomials is hypercontractive, Ann. of Math. (2) 174 (2011), no. 1, 485-497.

[11] A. Defant, D. García, and M. Maestre, Bohr's power series theorem and local Banach space theory, J. Reine Angew. Math. 557 (2003), 173-197.

[12] A. Defant, D. García, M. Maestre, and P. Sevilla-Peris, Bohr's strips for Dirichlet series in Banach spaces. part 2, Funct. Approx. Comment. Math. 44 (2011), no. part 2, 165-189.

[13] A. Defant, M. Maestre, and C. Prengel, The arithmetic Bohr radius, Q. J. Math. 59 (2008), no. 2, 189-205.

[14] A. Defant, M. Maestre, and U. Schwarting, Bohr radii of vector valued holomorphic functions, Adv. Math. 231 (2012), no. 5, 2837-2857. 
[15] A. Defant and P. Sevilla-Peris, A new multilinear insight on Littlewood's 4/3-inequality, J. Funct. Anal. 256 (2009), no. 5, 1642-1664.

[16] D. Diniz, G. A. Muñoz-Fernández, D. Pellegrino, and J. B. Seoane-Sepúlveda, The asymptotic growth of the constants in the Bohnenblust-Hille inequality is optimal, J. Funct. Anal. 263 (2012), no. 2, 415-428.

[17] D. Diniz, G. A. Muñoz-Fernández, D. Pellegrino, and J. B. Seoane-Sepúlveda, Lower bounds for the constants in the Bohnenblust-Hille inequality: the case of real scalars, Proc. Amer. Math. Soc. 142 (2014), no. 2, 575-580.

[18] B. C. Grecu, G. A. Muñoz-Fernández, and J. B. Seoane-Sepúlveda, Unconditional constants and polynomial inequalities, J. Approx. Theory 161 (2009), no. 2, 706-722.

[19] J. E. Littlewood, On bounded bilinear forms in an infinite number of variables, Quart. J. Math. Oxford 1 (1930), 164-174.

[20] A. Montanaro, Some applications of hypercontractive inequalities in quantum information theory, J. Math. Physics 53 (2012).

[21] G. A. Muñoz-Fernández, D. Pellegrino, and J. B. Seoane-Sepúlveda, Estimates for the asymptotic behaviour of the constants in the Bohnenblust-Hille inequality, Linear Multilinear Algebra 60 (2012), no. 5, 573-582.

[22] D. Núñez-Alarcón, A note on the polynomial Bohnenblust-Hille inequality, J. Math. Anal. Appl. 407 (2013), no. 1, 179-181.

[23] D. Nuñez-Alarcón, D. Pellegrino, and J. B. Seoane-Sepúlveda, On the Bohnenblust-Hille inequality and a variant of Littlewood's 4/3 inequality, J. Funct. Anal. 264 (2013), 326-336.

[24] D. Nuñez-Alarcón, D. Pellegrino, J. B. Seoane-Sepúlveda, and D. M. Serrano-Rodríguez, There exist multilinear Bohnenblust-Hille constants $\left(C_{n}\right)_{n=1}^{\infty}$ with $\lim _{n \rightarrow \infty}\left(C_{n+1}-C_{n}\right)=0$, J. Funct. Anal. 264 (2013), no. 2, 429-463.

[25] D. Pellegrino and J. B. Seoane-Sepúlveda, New upper bounds for the constants in the Bohnenblust-Hille inequality, J. Math. Anal. Appl. 386 (2012), no. 1, 300-307.

[26] K. Seip, Estimates for Dirichlet polynomials, EMS Lecturer, CRM (20), http://www.euro-mathsoc.eu/system/files/Seip_CRM.pdf.

[27] J. B. Seoane-Sepúlveda, Sharp generalizations of the multilinear Bohnenblust-Hille inequality, Oberwolfach Reports (European Mathematical Society) 11 (2014), no. 1, 378-381, DOI 10.4171/OWR/2014/06.

Departamento de Análisis Matemático,

Facultad de Ciencias Matemáticas,

Plaza de Ciencias 3,

Universidad Complutense de Madrid,

MADRID, 28040, SPAIN.

E-mail address: pablo_jimenez@mat.ucm.es

Departamento de Análisis Matemático, Facultad de Ciencias Matemáticas,

Plaza de Ciencias 3,

Universidad Complutense de Madrid,

MADRID, 28040, SPAIN.

E-mail address: gustavo_fernandez@mat.ucm.es

Instituto Universitario de Matemática Pura y Aplicada,

Universitat Politècnica de VALÈnCia,

46022, VALÈnCIA, SPAIN.

E-mail address: mamuar1@posgrado.upv.es

Departamento de Análisis Matemático,

Facultad de Ciencias Matemáticas,

Plaza de Ciencias 3,

Universidad Complutense de Madrid,

MADRID, 28040, SPAIN.

E-mail address: jseoane@mat.ucm.es 